\title{
Virtual Reality and Virtual Reality System Components
}

\author{
Oluleke Bamodu ${ }^{1,2, a}$ and Xuming $\mathrm{Ye}^{1, \mathrm{~b}}$ \\ ${ }^{1}$ College of Mechanical Engineering, Shenyang University, Shenyang, China \\ ${ }^{2}$ Faculty of Computing, Engineering and Technology, Staffordshire University, United Kingdom \\ aindomitablejnr@engineer.com, byxm1116888@163.com
}

Keywords: virtual reality, VR, virtual reality system, hardware, software, VR engine.

\begin{abstract}
This paper is a synoptic review of virtual reality, its features, types, and virtual reality systems, as well as the elements of the VR system hardware and software, which are essential components of virtual reality systems.
\end{abstract}

\section{Introduction}

Applications of Virtual Reality (VR) have and continue to increase over the last couple of years. This is partly due to its usefulness in many fields and as a result of the attention given to it by the media. This trend is expected to continue in the future with the advancement of technology in areas like computer graphics, computer vision, controls, image processing and other technology-affiliated components. This paper aims to dissect the nature, the role, the component, the application and applicability of the virtual reality system, as well as, explore briefly the characteristics and use of VR system hardware and software.

\section{Definition of Virtual Reality}

Defining Virtual Reality can prove to be a difficult task because there is no standard definition for it. It is said to be an oxymoron, as it is referred to by some school of thought as "reality that does not exist" [1]. Many different fields have different meaning associated to it, while some people have even misused the term in many cases. However, [2] rightly defines VR as "a medium composed of interactive computer simulations...giving the feeling of being immersed...”, while [3] expressed it better and finely as "a high end Human-Machine Interface, that combine technologies such as computer graphics, image processing, pattern recognition, artificial intelligence, networking, sound systems and others to produce computer simulation and interaction, which gives the feeling of being present through multiple synthetic feedback sent to sensorial channels like virtual, aural, haptic and others” [3]. Many other names are being used interchangeably with VR; these include Virtual Environment, Artificial Reality, Virtual Worlds, Artificial Worlds, and Cyberspace.

\section{Features of Virtual Reality}

Inferring from the definitions above, it would not be wrong to say that the quality of any VR system is determined by certain features. For the purpose of this review, focus will be on three (3) features, termed the 3 'I' or triangle. [4,5]. The 3'I' stands for Immersion, Interaction and Imagination

Imagination, otherwise known as presence, is the feeling of being present or being a part of the computer generated world. This is as a result of the stimulation of the human senses (visual, aural, haptic, smell etc) by the system.

Interaction is a means of communicating with the system, but unlike the traditional Human-Computer Interaction which uses 1-2 dimensional (1D, 2D) means, like mouse, keyboard or keypad, interaction in VR is usually through 3 dimensional (3D) means, like space ball and head-mounted device (HMD). Some features for interaction in VR systems are effectiveness, real time reaction and human participation. 
Imagination can be seen as the thought of the system designer to execute a particular goal. With the applicability of components of the VR system for complex problem solving in diverse fields, its use as a more efficient and effective means of expressing ideas than the traditional 2D drawing or text explanation, cannot be disputed.

\section{Types of Virtual Reality Systems}

VR systems can be classified into 3 major categories. These are, non-immersive, immersive and semi-immersive, based on one of the important features of VR, which is immersion and the type of interfaces or components utilized in the system.

Non-Immersive VR system, also called Desktop VR system, Fish tank or Window on World system is the least immersive and least expensive of the VR systems, as it requires the least sophisticated components. It allows users to interact with a 3D environment through a stereo display monitor and glasses, other common components include space ball, keyboard and data gloves. Its application areas include modeling and CAD systems [6].

Immersive VR system on the other hand is the most expensive and gives the highest level of immersion; its components include HMD, tracking devices, data gloves and others, which encompass the user with computer generated 3D animation that give the user the feeling of being part of the virtual environment. One of its applications is in virtual walk-through of buildings.

Semi-Immersive VR system, also called hybrid systems [1] or augmented reality system, provides high level of immersion, while keeping the simplicity of the desktop VR or utilizing some physical model. Example of such system includes the CAVE (Cave Automatic Virtual Environment) and an application is the driving simulator [7].

Distributed-VR also called Networked-VR is a new category of VR system, which exists as a result of rapid development of internet. Its goal is to remove the problem of distance, allowing people from many different locations to participate and interact in the same virtual world through the help of the internet and other networks. A traditional application of this is the SIMNET which is a real time distributed simulation developed by the US military and used for combat trainings [3, 4].

\section{Components of Virtual Reality Systems}

A VR system is made up of 2 major subsystems, the hardware and software. The hardware can be further divided into computer or VR engine and I/O devices, while the software can be divided into application software and database as illustrated below.

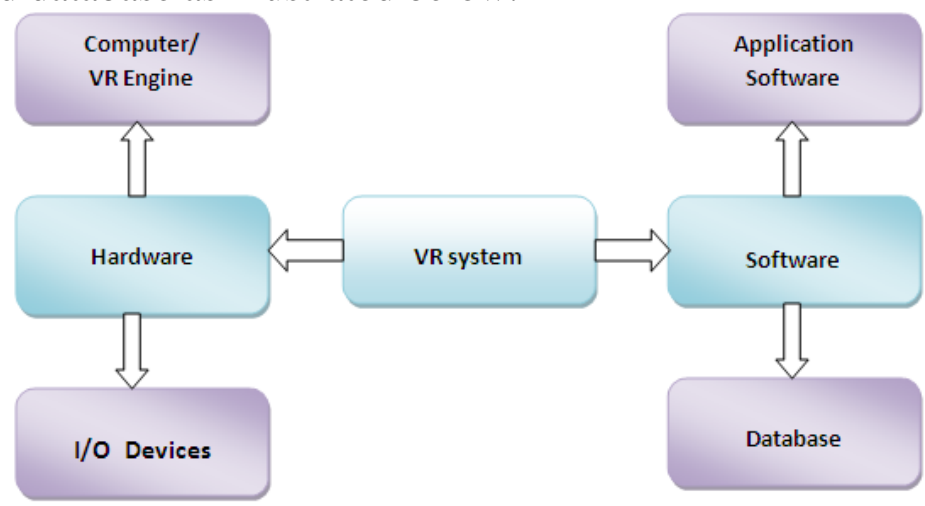

Fig. 1

\section{Virtual Reality System Hardware}

The major components of the hardware are the VR engine or computer system, input devices and output devices shown in fig. 2 below. 


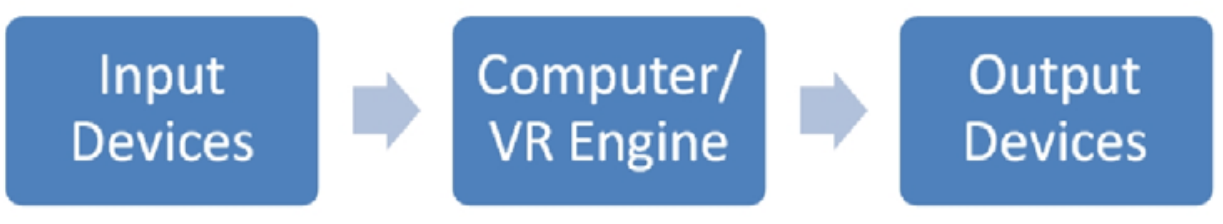

Fig. 2

Input Devices. The input devices are the means by which the user interacts with the virtual world. They send signals to the system about the action of the user, so as to provide appropriate reactions back to the user through the output devices in real time. They can be classified into tracking device, point input device, bio-controllers and voice device.

Tracking devices sometimes referred to as position sensors, are used in tracking the position of the user [1], and they include, electromagnetic, ultrasonic, optical, mechanical and gyroscopic sensors, data gloves, neural and bio or muscular controllers [2].

Examples of point-input devices include 6DOF mouse and force or space ball. Their technology is an adaptation of the normal mouse with extended functions and capability for 3D.

Voice communication is a common way of interaction among humans. So it feels natural to incorporate it into a VR system. Voice recognition or processing software can be used in accomplishing this.

VR Engine. In VR systems, the VR engine or computer system has to be selected according to the requirement of the application. Graphic display and image generation are some of the most important factors and time consuming task in a VR system. The choice of the VE engine depends on the application field, user, I/O devices, level of immersion and the graphic output required, since it is responsible for calculating and generating graphical models, object rendering, lighting, mapping, texturing, simulation and display in real-time. The computer also handles the interaction with users and serves as an interface with the $\mathrm{I} / \mathrm{O}$ devices.

A major factor to consider when selecting the VR engine is the processing power of the computer, and the computer processing power is the amount of senses (graphical, sound, haptic, etc) that can be rendered in a particular time frame as pointed. The VR engine is required to recalculate the virtual environment approximately every 33ms and produce real time simulation of more than 24fps [4], furthermore, the associated graphic engine should be capable of producing stereoscopic vision. The VR engine could be a standard PC with more processing power and a powerful graphics accelerator or distributed computer systems interconnected through high speed communication network.

Output Devices. The output devices get feedback from the VR engine and pass it on to the users through the corresponding output devices to stimulate the senses. The possible classifications of output devices based on the senses are: graphics (visual), audio (aural), haptic (contact or force), smell and taste. Of these, the first 3 are frequently used in VR systems, while smell and taste are still uncommon.

Two possible common options for the graphics are the stereo display monitor, and the HMD which provides a higher level of immersion. In the HMD, the two independent views produced are interpreted by the brain to provide a $3 \mathrm{D}$ view of the virtual world.

Audio or sound is an important channel in VR; its importance is only surpassed by that of visual. $3 \mathrm{D}$ sound can be used in producing different sounds from different location to make the VR application more realistic.

Haptic is used to allow the user feel virtual objects. This can be achieved through electronic signals or mechanical devices.

\section{Virtual Reality System Software and Tools}

Virtual reality system software is a colletion of tools and software for designing, developing and maintaining virtual environments and the database where the information is stored. The tools can be classified into modeling tools and development tools. 
VR Modeling Tools. There are many modeling tools available for VR designing, the most common ones are , 3ds Max, Maya and Creator. Engineering specific applications might use software like CATIA, Pro/E, Solidworks, UG, etc.

VR Development Tools. VR is a complex and integrative technology that borrows from many other technologies, such as real time 3D computer graphics, tracking technology, sound processing, and haptic technology, among others, therefore software development flexibility and real time interaction is needed. Starting the development of a VR system from the basic codes in $\mathrm{C} / \mathrm{C}++$, Java, OpenGL, etc, requires a large amount of work and such system reliability is usually low, therefore VR development tools are used.

Careful consideration is needed in choosing VR development tools due to the difference in flexibility provided by different software packages as related to model input available, interface compatibility, file format, animation ease, collision detection, supported I/O devices and support community available to the users.

VR development tools used in VR content creation include, virtual world authoring tools, VR toolkits/software development kits (SDK) and application program interfaces (APIs). But it is not uncommon to find that some APIs are also toolkits, like OpenGL optimizer and Java 3D API [1, 6].

\section{Applications of Virtual Reality}

VR has found vast applications in many fields due to its characteristics and the benefits it provide in solving complex real-world problems. Some of the application areas include: Architecture, Arts, Business, Design and Planning, Education and Training, Entertainment, Manufacturing, Medical and Scientific Visualization.

In manufacturing, VR is used to remove limitations in virtualization and interaction associated with traditional 3D CAD/CAM systems through virtual manufacturing. Virtual manufacturing is virtual product design, modeling, simulation, assembly, testing and analysis for error before physical prototypes are built to reduce development time and avoid wasteful costs [9].

\section{References}

[1] T.H. Dani and G. Rajit, Virtual Reality - A New Technology for the Mechanical Engineer In: Mechanical Engineers’ Handbook, 2nd ed., ed. Meyer Kutz. John Wiley \& Sons, Inc., New York, 1998, pp. 319-327

[2] A. B Craig, R. S William and D. W Jeffrey, Developing Virtual Reality Applications: Foundations of Effective Design, Morgan Kaufmann Publishers, Burlington, 2009

[3] C. H Zhuang and P. WANG, Virtual Reality Technology and Applications (in Chinese). Publishing House of Electronics Industry Beijing, 2010.

[4] G.C Burdea and C. Philippe, Virtual Reality Technology, 2nd ed. John Wiley \& Sons, Inc. New York, 2003.

[5] W. Shen and W. Q Zeng, Virtual Reality Technology (in Chinese), Tsinghua University Press, Beijing, 2009.

[6] J. Isdale, What Is Virtual Reality? A Web-Based Introduction, Version 4, Information on http://vr.isdale.com/WhatIsVR/frames/WhatIsVR4.1.html

[7] J. M Blackedge, M. Barrett and E. Coyle, Using Virtual Reality to Enhance Electrical Safety and Design in the Built Environment, Paper 17, 2010. Information on

http://arrow.dit.ie/engscheleart2/17

[8] X. Q Hu, Virtual Reality Technology Fundamentals and Applications (in Chinese). Beijing University of Posts and Telecommunication Press, Beijing 2009

[9] O. Bamodu and X. Ye, Virtual Manufacturing and Components of Virtual Reality,in press, 2012 